\title{
Robustness of first-order phase transitions in one-dimensional long-range contact processes
}

\author{
Carlos E. Fiore and Mário J. de Oliveira \\ Departamento de Fúsica, Universidade Federal do Paraná \\ Caixa Postal 19044, 81531-000 Curitiba, Paraná, Brazil and \\ Instituto de Física, Universidade de São Paulo \\ Caixa Postal 66318, 05315-970 São Paulo, Brazil
}

(Dated: September 28, 2018)

\begin{abstract}
It has been proposed (Phys. Rev. E 71, 026121 (2005)) that unlike the short range contact process, a long-range counterpart may lead to the existence a discontinuous phase transition in one dimension. Aiming at exploring such link, here we investigate thoroughly a family of longrange contact processes. They are introduced through the transition rate $1+a \ell^{-\sigma}$, where $\ell$ is the length of inactive islands surrounding particles. In the former approach we reconsider the original model (called $\sigma$-contact process), by considering distinct mechanisms of weakening the long-range interaction toward the short-range limit. Second, we study the effect of different rules, including creation and annihilation by clusters of particles and distinct versions with infinitely many absorbing states. Our results show that all examples presenting a single absorbing state, a discontinuous transition is possible for small $\sigma$. On the other hand, the presence of infinite absorbing states leads to distinct scenario depending on the interactions at the frontier of inactive sites.

PACS numbers: 05.70.Ln, 05.50.+q, 05.65.+b
\end{abstract}

\section{INTRODUCTION}

Nonequilibrium phase transitions into absorbing states describe a large sort of phenomena including chemical reactions, disease spreading, competition between species, wetting processes, calcium dynamics and others [1, 2]. Due the absence of equilibrium analogous, they are essential in the establishment of the main ingredients required for the emergence of phase transitions and critical behavior.

The contact process (CP) [3] is probably the best example yielding an absorbing phase transition. Particles are created catalytically, but are spontaneously annihilated. It presents a set of critical exponents belonging to the directed percolation (DP) universality class. The DP conjecture [4] embraces not only the CP, but also generic absorbing phase transitions with no extra symmetries and conservation laws. Such examples are reactiondiffusion processes, cellular-automata models and even continuous descriptions with multiplicative noise [5, 6]. More recently it has been observed experimentally in turbulent nematic liquid crystals [7].

Differently from the continuous case, one-dimensional discontinuous absorbing transitions has been much less observed. Except in special cases [8 10], its manifestation in short range systems have been the subject of a longstanding controversy [11 15]. The absence of a discontinuous transition would stem from the suppression of compact clusters coming from the large fluctuations in one dimension. On the other hand, by increasing the dimensionality, the fluctuations are less relevant and the formation of compact clusters becomes possible.

Long-range interactions have been proposed like more realistic descriptions in different nonequilibrium phenomena, when compared with their short-range counterparts. Some examples of systems presenting long-range interac- tions include wetting phenomena, spreading of diseases over long distances and others [16 18]. As an effect of long-range interactions, the absorbing transition may deviate from the original DP case and belong to the different universality classes [19 24]. Other remarkable difference concerns the possibility of stabilizing compact clusters in one dimension. Ginelli et al. 25] introduced the $\sigma$-contact process, in which particles are created and annihilated like the usual short-range $\mathrm{CP}$, but the activation rate depends the length $\ell$ of the island of inactive sites according to expression $1+a \ell^{-\sigma}$. They found that for $0<\sigma<1$ the interactions are effectively long-range and the phase transition becomes discontinuous. On the other hand, for $\sigma>1$ the long-range parameter play not relevant role and the phase transition remains secondorder (similar to its short-range version).

Despite the study of the $\sigma-\mathrm{CP}$ under different methodologies [25, 26] some aspects have not been addressed so far. Is it present a first-order transition in the limit of extreme weak $(a<<1)$ long-range interactions? Does the competition with short range interactions become the system still able to suppress fluctuations that destabilize compact clusters? Is the phase coexistence maintained by changing the interaction rules? Does the existence of infinitely absorbing states influence the order of transition?

Aiming to answer above queries, in this paper we investigate thoroughly a family of one-dimensional longrange contact models. First, we reconsider the $\sigma-\mathrm{CP}$ by weakening sufficiently the long-range interaction toward the short-range limit and further by introducing an effective competition with short-range interactions. Although the emergence of a discontinuous transition is expected not depending on the parameter $a$ [25], a quite interesting point would concern the stabilization of compact clusters over extreme small long-range interactions 
(thus close to the short-range regime). In such cases the long-range should act as a small (but relevant) perturbation. Second, we consider the effect of different interaction rules, including creation and annihilation in the presence of clusters of particles (instead of one particle case) and infinitely many absorbing states. These models are long-range versions of the named pair-creation $\mathrm{CP}$, pair-annihilation (PAM), triplet-annihilation models (TAM) and the pair contact process (PCP), respectively [11, 27, 28]. All models will be studied over mean-field approximations and extensive numerical simulations in the constant rate (ordinary) [1] and the constant particle number (conserved) ensembles [26, 29 32]. Our results show that for all systems with a single absorbing state the occurrence of discontinuous transitions is held by decreasing $\sigma$. For the long-range PCP, on the other hand, different scenario are possible. By measuring the length $\ell$ between extreme pairs of particles, the transition becomes first-order for low $\sigma$. On the other hand, when $\ell$ is the distance between a pair and the nearest particle surrounding the inactive island, the transition is always continuous.

This paper is organized as follows: In Sec. II we described all methods, in Sec. III we present the models and numerical results and finally conclusions are showed in Sec. IV.

\section{CONSTANT RATE AND CONSERVED ENSEMBLES}

The one-dimensional contact process is defined in a chain of $L$ sites where each site $i$ is attached by a twostate occupation variable $\eta_{i}$ reading $\eta_{i}=0$ or 1 , according to whether it is empty or occupied, respectively. Interaction rules are composed of creation and annihilation of particles, represented by transition rates $\omega_{i}^{c}$ and $\omega_{i}^{a}$. Particles are created in empty active sites and are spontaneously annihilated.

Systems will be studied in the constant rate (ordinary) and in the constant particle number (conserved) ensembles [26, 29, 31, 32]. In the former case the control parameter (the creation or annihilation rates) are held fixed but the total particle number $\bar{n}$ fluctuates. It is described by the total transition rate $w_{i}$

$$
w_{i}=\omega_{i}^{c}+\alpha \omega_{i}^{a}
$$

where $\alpha$ denotes the strength of the annihilation rate. For low $\alpha$, phase is active, in which particles are continuously created and annihilated. By increasing $\alpha$, a phase transition into an absorbing state takes place. Except the PCP, the absorbing state is characterized by a full empty lattice. For the PCP, any configuration devoid of pairs is absorbing.

The transition point and the nature of transition can be precisely identified by performing spreading simulations 1]. Starting from an initial seed, it consists of determining the time evolution of appropriate quantities, such as the survival probability $P_{s}(t)$, the total number of particles $N(t)$ and the mean square spreading $R^{2}(t)$.

At the emergence of phase transition, these quantities follow algebraic behaviors given by

$$
P_{s}(t) \sim t^{-\delta}, \quad N(t) \sim t^{\eta} \quad \text { and } \quad R^{2}(t) \sim t^{2 / z},
$$

where $\delta, \eta$ and $z$ are their associated dynamic critical exponents. For second-order DP phase transitions, they read $\delta=0.159464(6), \eta=0.313686(8)$ and $z=$ $1.580745(10)$ [6]. In a discontinuous transition, despite the order parameter gap, they also present algebraic behaviors with critical exponents given by $\delta=1 / 2, \eta=0$ and $z=1$, which is compatible with the Glauber-Ising (GI) model [6].

Thus, spreading simulations provides not only locating the transition point, but also to classify the order of transition. The change in the order of transition will be characterized by an alteration in the critical exponents. Off the transition regime, above dynamic quantities deviate from the power law behaviors.

For systems with infinitely many absorbing states [33], spreading experiments become particularly hard to be used. In particular, the dynamic exponents $\delta, \eta$ and $z$ are strongly dependent on the initial conditions, presenting non-universal values [33 36]. A simpler procedure for locating the critical point in such cases is to study the order-parameter decay starting from a fully occupied initial condition. Unlike above exponents, $\theta$ does not depend on the initial configuration. One expects $\phi$ behaves as $\phi \sim t^{-\theta}$ at the critical point, where $\theta=\delta$. Conversely, we should expect a non power-law behavior at a discontinuous transition. Since alternative methods (e.g hysteretic ones) can not be used for systems with absorbing states, another strategy is required. In particular, we have calculated the probability distribution (in the steady regime) by considering different initial configurations. A bimodal distribution reveals the discontinuous transition.

In the constant rate ensemble, the control parameter is the total particle number $n$. Particles are created as in the ordinary case, but instead of creating new particles, a cluster of $k$ sites leaves its place and jumps to $k$ active sites. One may define the conserved ensemble as a $2 k$ site process, in which creation and annihilation occurs simultaneously according to the following transition [32]

$$
w=\omega_{i}^{a} \underbrace{\omega_{j}^{c} \omega_{l}^{c} \ldots \omega_{m}^{c}}_{k-\text { creation }} .
$$

It has been shown [31, 32] that in the thermodynamic limit above dynamics is equivalent to that studied in the constant rate ensemble. The parameter $\bar{\alpha}$ fluctuates and is calculated through expression

$$
\bar{\alpha}=\frac{\left\langle\omega_{j}^{c}\right\rangle_{c}}{k\left\langle\omega_{i}^{a}\right\rangle_{c}}
$$

where $\langle\ldots\rangle_{c}$ denotes a generic average evaluated over the conserved ensemble. An immediate advantage concerns 
its simplicity for locating the transition point. In this case, by considering the system constrained in the subcritical regime, e. g, a finite number of particles placed in an infinite lattice, the addition of particles drives the system toward the transition point $\alpha_{0}$ according to the expression [26, 29, 32, 37],

$$
\bar{\alpha}-\alpha_{0} \sim n^{-1} .
$$

Thus, from the above, the transition point is obtained by a linear extrapolation in $1 / n$. Other advantage refers the classification of the phase transition, obtained by measuring the particle structures for different $n$. For secondorder transitions, the clusters are fractals [38], whereas they become compact in a discontinuous transition. Being $R$ the mean distance of particles located at extremities of the system, we have that [1, 26, 32]

$$
R \sim n^{1 / d_{F}},
$$

where $d_{F}$ is the fractal dimension. For one-dimensional systems belonging to the DP universality class $d_{F}$ reads $0.74792 \ldots$, whereas at the phase coexistence it is the proper euclidean dimension $d=1$ (consistent to the existence of compact clusters). The above values are related with dynamic exponents through expression $d_{F}=$ $2(\eta+\delta) / z$.

\section{MEAN FIELD APPROXIMATION}

Before performing simulations we have analyzed the models by means of a cluster approximation at the level of two nearest neighbor sites [26]. In this case, the system is described by the one-site probabilities $P(0)$ and $P(1)$ and the two-site probabilities $P(11), P(10), P(01)$ and $P(11)$. However, only two of them are independent. The generic probability of a string of $\ell$ consecutive sites is approximated by

$P\left(\eta_{1}, \eta_{2}, \eta_{3}, \ldots, \eta_{\ell}\right)=\frac{P\left(\eta_{1}, \eta_{2}\right) P\left(\eta_{2}, \eta_{3}\right) \ldots P\left(\eta_{\ell-1}, \eta_{\ell}\right)}{P\left(\eta_{2}\right) P\left(\eta_{3}\right) \ldots P\left(\eta_{\ell-1}\right)}$.

By choosing $P(1)=\left\langle\eta_{i}\right\rangle$ and $P(11)=\left\langle\eta_{i} \eta_{i+1}\right\rangle$ and taking account the translation invariance, their evolution equations read

$$
\frac{d}{d t}\left\langle\eta_{i}\right\rangle=\left\langle\left(\bar{\eta}_{i}-\eta_{i}\right) w_{i}(\eta)\right\rangle
$$

and

$\frac{d}{d t}\left\langle\eta_{i} \eta_{i+1}\right\rangle=\left\langle\left(\bar{\eta}_{i}-\eta_{i}\right) \eta_{i+1} w_{i}(\eta)\right\rangle+\left\langle\eta_{i}\left(\bar{\eta}_{i+1}-\eta_{i+1}\right) w_{i+1}(\eta)\right\rangle$,

where $\bar{\eta}_{i} \equiv 1-\eta_{i}$. By using the approximation (8), we get a set of two closed equations for $P(1)=\rho$ and $P(11)=\phi$. At the stationary state we found for all models studied a general structure in the relation for $\alpha$ vs $\rho$ given by

$$
\alpha \rho=\alpha_{c} \rho+A \rho^{2}
$$

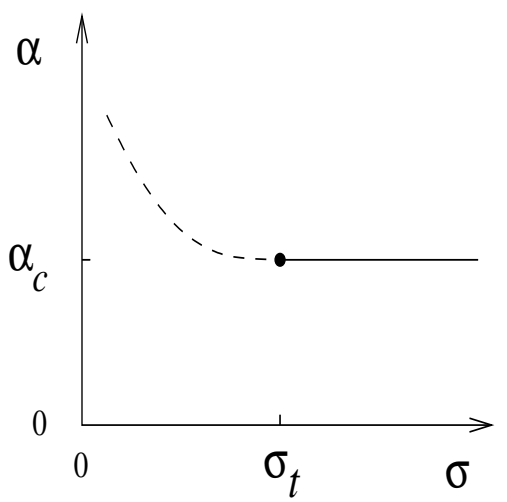

FIG. 1: The pair mean-field approximation phase diagram in the space $\alpha$ versus $\sigma$. Continuous and dashed lines denote second and first order phase transitions, respectively and the full circle denotes the tricritical point.

up to order $\rho^{2}$, where $\alpha_{c}$ is a numerical constant and $A$ depends on the other parameters but not on $\alpha$. From this equation it follows that a critical line occurs at $\alpha=\alpha_{c}$ and $A<0$. When $A>0$, the transition becomes firstorder and a tricritical point occurs at $A=0$. The phase diagram is of the type shown in Fig. 1.

\section{NUMERICAL RESULTS}

Except for the PCP model, numerical simulations will be performed for large system sizes $\left(L=2^{16}\right)$ and periodic boundary conditions. In the conserved case, MC simulations are started by constraining the system in the subcritical (absorbing) regime. In practice, it is done by taking finite $n$ 's in a large $L$ and check whether a particle touches the border. If a particle reaches the border, we increase $L$. By simulating distinct $n$ 's (with $\bar{\alpha}$ computed from Eq. (5) ), the transition point $\alpha_{0}$ is obtained by means of a linear extrapolation in $1 / n$. The nature of phase transition is identified by calculating the fractal dimension, measured from the dependence of $R$ on $n$. Further, we check above results by performing epidemic simulations starting from an initial seed in which the transition point $\alpha_{0}$ is located by identifying algebraic behaviors for $N$ and $P_{s}$. Their corresponding dynamic exponents $\eta$ and $\delta$ classifies the order of transition.

\section{A. Long-range contact process $(\sigma-\mathbf{C P})$}

In the usual $\mathrm{CP}$, particles are created in empty sites surrounded by at least one particle and are spontaneously annihilated. It is defined by the transition rates

$$
\omega_{i}^{c}=\frac{1}{2}\left(1-\eta_{i}\right) \sum_{\delta} \eta_{i+\delta}
$$



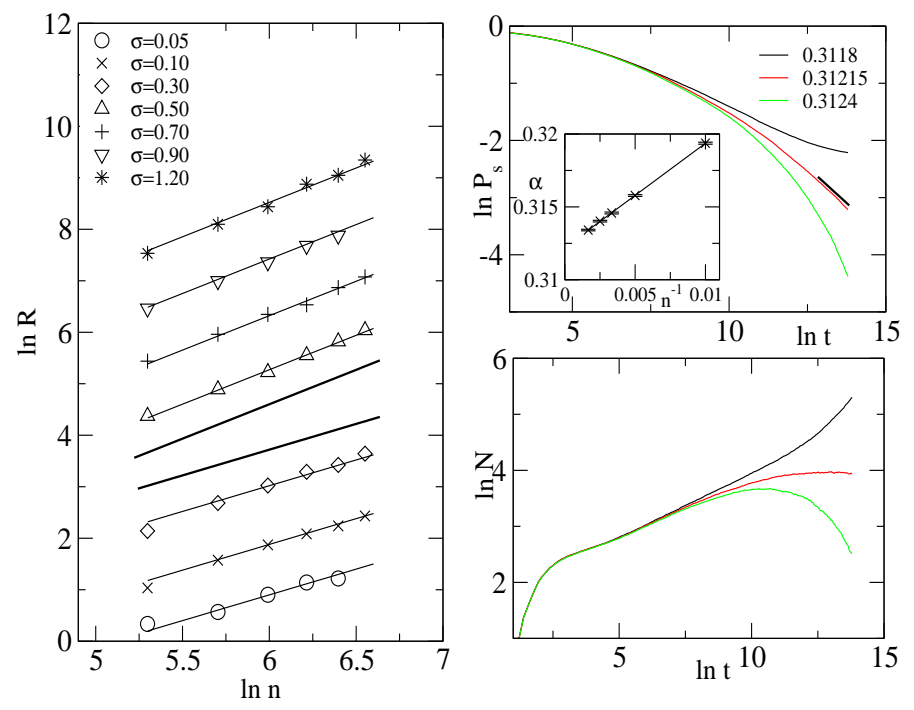

FIG. 2: In the left, log-log plot of the average cluster size $R$ versus the total number of particles $n$ for several values of $\sigma$ for $a=0.05$. The up and down predicted curves have slopes 1.33704 and 1 , respectively. The data points have been shifted in order to avoid overlapping. In the right, log-log plot of $P_{s}$ and $N$ for distinct values of $\alpha$ for $\sigma=0.1$. The predicted asymptotic slopes are consistent with GI values. In the inset parameter $\bar{\alpha}$ versus $n^{-1}$ in the conserved ensemble.

and

$$
\omega_{i}^{a}=\eta_{i},
$$

for particle creation and annihilation, respectively. In the $\sigma-\mathrm{CP}$ the creation rate is replaced by the following expression

$$
\begin{aligned}
& \omega_{i}^{c}=\frac{1}{2} \sum_{\ell=1}^{\infty}\left(1+\frac{a}{\ell^{\sigma}}\right) \eta_{i-1} \bar{\eta}_{i} \bar{\eta}_{i+1} \ldots \bar{\eta}_{i+\ell-1} \eta_{i+\ell} \\
& +\frac{1}{2} \sum_{\ell=1}^{\infty}\left(1+\frac{a}{\ell^{\sigma}}\right) \eta_{i+1} \bar{\eta}_{i} \bar{\eta}_{i-1} \ldots \bar{\eta}_{i-\ell+1} \eta_{i-\ell},
\end{aligned}
$$

which depends on $\ell, a$ and $\sigma$ and $\bar{\eta}_{i} \equiv 1-\eta_{i}$. For $a=0$, one recovers the original short-range $\mathrm{CP}$, whose secondorder phase transition occurs at $\alpha_{c}=0.303227 \ldots$ [1].

Here we have weakened the long-range interaction toward the short-range limit, in order to see if a phase coexistence still exists for $a<<1$. In Fig. 2, we show results for $a=0.05$. Note that the fractal dimension changes (from 0.75 to 1 ) for $\sigma<\sigma_{t}=0.4(1)$, consistent with the emergence of a discontinuous transition for smaller $\sigma$. Spreading experiments, showed in the right part for $\sigma=0.1$, confirms such conclusion. At $\alpha_{0}=0.31215(5)$, both quantities $P_{s}$ and $N$ present algebraic behaviors consistent with exponents $\delta=1 / 2$ and $\eta=0$, respectively. The above estimate agrees very well with the conserved ensemble result $\alpha_{0}=0.31221(3)$. In addition, we have also studied the possibility of discontinuous transitions for more extreme cases. For $a=0.01$
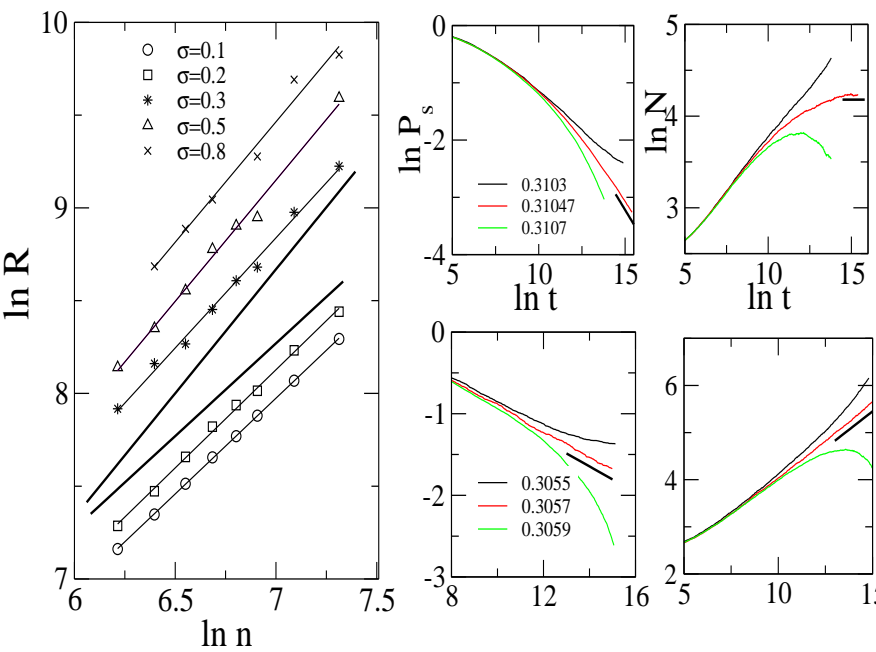

FIG. 3: In the left, log-log plot of the average cluster size $R$ versus the total number of particles $n$ for several values of $\sigma$ at the subcritical regime. The up and down straight lines have slopes 1.33704 and 1, respectively. The data points have been shifted in order to avoid overlapping. In the right, from top to bottom, log-log plot of the time evolution of $P_{s}$ and $N$ for distinct values of $\alpha$ for $\sigma=0.1$ (top) and $\sigma=0.3$ (bottom), respectively. The predicted asymptotic slopes are consistent with GI (top) and DP (bottom) values.

and $\sigma=0.005$, the transition is first-order, yielding at $\alpha_{0}=0.30572(3)$, a value rather close to the short-range case 0.303227 . For completeness, we have considered the opposite case, e.g. the occurrence of discontinuous transition for larger $a$ 's. Our results (not shown) support that the first-order transition line moves toward larger $\sigma$ 's. For example, for $a=5$ and $\sigma=1.2$, the transition is first-order yielding at $\alpha_{c}=0.439655(5)$. The crossover occurs at $\sigma_{t}=1.3(1)$, which is larger than $\sigma_{t}=1.0(1)$ for $a=2$.

Above conclusions are also predicted by pair mean-field results. It gives a critical line at the value $\alpha_{c}=1 / 2$ and the tricritical point occurring at

$$
\zeta\left(\sigma_{t}\right)=\frac{1+a}{a},
$$

where $\zeta(\sigma)$ is the Riemann zeta function defined by $\zeta(\sigma)=\sum_{k=1}^{\infty} k^{-\sigma}$. From the above, it follows that $\sigma_{t}>1$, in accordance with numerical results for larger $a$ but not for sufficient small $a$ 's.

Further, we introduce the competition with shortrange interactions. This will be accomplished by performing short and long-range processes probabilities $p$ and $1-p$, respectively. For low $p$, one expects a qualitative behavior similar to the full long-range case, whereas for extreme large $p$ if a change in the transition occurs, it should manifest for sufficient low $\sigma$. In Fig. 3 we examine the phase transitions for $p=0.98$ and different $\sigma$. As in the previous case, the system structure also changes by decreasing $\sigma$ and clusters become compact for $\sigma<0.3$. This is also checked by comparing the time evolution of 


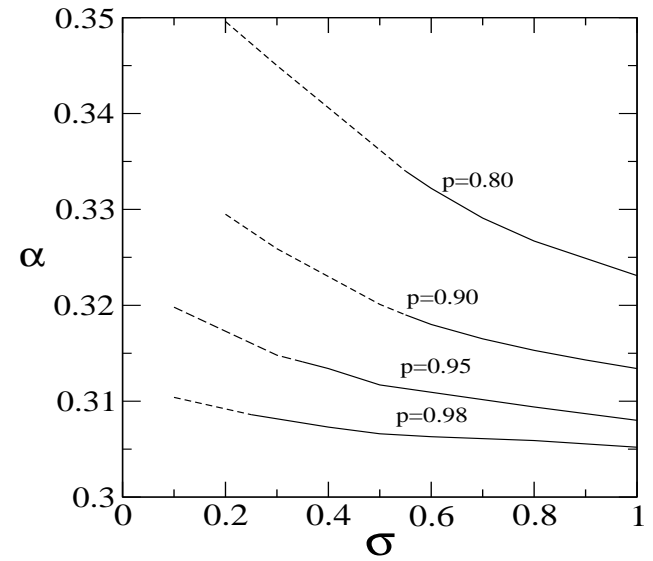

FIG. 4: Parameter $\alpha$ versus $\sigma$ for different values of $p$. Continuous and dashed lines denote second and first-order transitions, respectively. For each $p$, absorbing and active phases are located above and bellow of corresponding lines, respectively.

$P_{s}$ and $N$ for $\sigma=0.3$ and $\sigma=0.1$. At the transition points $\alpha_{c}=0.3057(1)$ and $\alpha_{0}=0.31045(5)$, above quantities present distinct algebraic behaviors consistent with the DP and GI exponents. In Fig. 4 we show the phase diagram for distinct (but large) values of $p$. As expected, the coexistence line move toward lower values of $\sigma$.

In summary, above "weakening" approaches show that it suffices a small long-range "perturbation" in the original $\mathrm{CP}$ to provoke a change in the order of transition.

\section{B. Long-range pair-creation, pair and triplet annihilation contact models}

Here we study the effect of distinct interaction rules in the long-range $\mathrm{CP}$. The first change, called $\sigma$-pair $\mathrm{CP}$, is similar to the $\sigma-\mathrm{CP}$ but new particles can be created only in empty sites surrounded by pairs of particles. The creation rate $\omega_{i}^{c}$ reads

$$
\begin{aligned}
& \omega_{i}^{c}=\frac{1}{2} \sum_{\ell=1}^{\infty}\left(1+\frac{a}{\ell^{\sigma}}\right) \eta_{i-2} \eta_{i-1} \bar{\eta}_{i} \bar{\eta}_{i+1} \ldots \bar{\eta}_{i+\ell-1} \eta_{i+\ell} \\
& +\frac{1}{2} \sum_{\ell=1}^{\infty}\left(1+\frac{a}{\ell^{\sigma}}\right) \eta_{i+2} \eta_{i+1} \bar{\eta}_{i} \bar{\eta}_{i-1} \ldots \bar{\eta}_{i-\ell+1} \eta_{i-\ell} .
\end{aligned}
$$

The limit $a=0$ corresponds to the short-range case, in which a DP phase transition yields at $\alpha_{c}=0.13397(4)$ [32]. In Fig. 5, we show the main results for distinct $\sigma$ 's and $a=2$. The transition is also continuous for $\sigma>1$ and becomes first-order for $0<\sigma<1$. However, as an effect of the creation in the presence of pairs, the cluster are somewhat more compact than the usual $\sigma-\mathrm{CP}$. For example, for $\sigma=0.5$ the cluster density $\rho=N / R$ (evaluated from the inverse of slopes of curves $R$ vs $n$ ) is

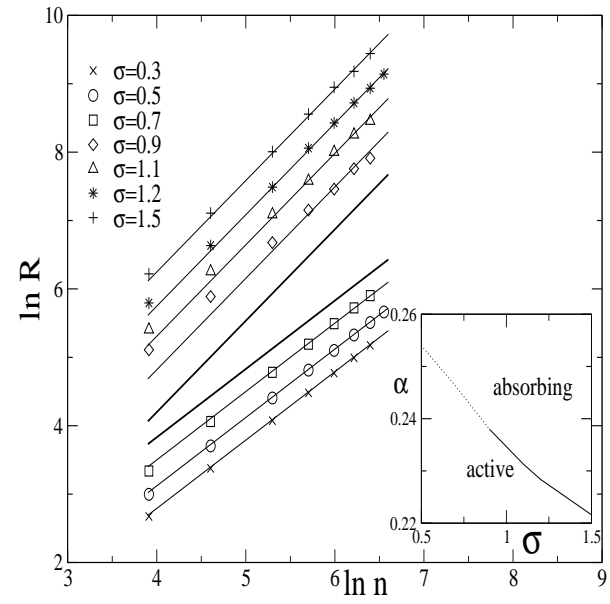

FIG. 5: Log-log plot of $R$ versus $n$ for the long-range paircreation CP for distinct values of $\sigma$. The up and down straight lines have slopes 1.33704 and 1 , respectively and they have been shifted in order to avoid overlapping. In the inset, we show its phase diagram. Continuous and dotted lines denote continuous and discontinuous transition, respectively.

about 0.78 for the $\sigma-\mathrm{CP}$, whereas it reads 0.81 for the $\sigma$-pair CP. However, the creation by pairs of particles is not sufficient effective for shifting the coexistence line for larger $\sigma$. In contrast with the $\sigma-\mathrm{CP}$, above results are not predicted by pair mean-field approximation, in which the phase transition is always first order. On the other hand, when $\sigma \rightarrow \infty$ the parameter $\alpha \rightarrow 1 / 4$, which is in accordance with the mean-field short-range case.

Next, we consider the opposite situation, in which particles are created like the above $\sigma-\mathrm{CP}$, but only pairs of particles are allowed to be annihilated. The annihilation rate $\omega_{i}^{a}$ reads

$$
\omega_{i}^{a}=\eta_{i} \eta_{i+1} .
$$

In the conserved ensemble, Eq. (44) is used for calculating for $\bar{\alpha}$ by taking $k=2$. For $a=0$ one recovers the original pair-annihilation contact model (PAM), in which a DP continuous phase transition yields at $\alpha_{c}=0.18622(3)$ [27, 32].

Like the $\sigma-\mathrm{CP}$, for $a=2$ the phase transition becomes first-order for $0<\sigma<1$. The crossover between continuous and discontinuous occurs between 0.8 and 1.1. In Fig. 6] we show the main results for distinct $\sigma$ 's. As an consequence of the pair annihilation, the compact clusters are less dense than the previous cases (e. g., for $\sigma=0.5$ the cluster density $\rho$ is about $2 / 3$ ).

Using Eq. (5) we have built the phase diagram shown in Fig. 7. Since $\left\langle\omega_{i}^{c}\right\rangle_{c}$ is proportional to $\ell^{-\sigma}$, it increases by decreasing $\sigma$. On the other hand, the average $\left\langle\omega_{i}^{a}\right\rangle_{c}$ also increases, as a result of more compact particle displacements. The competition between averages results in a net increase of $\alpha$ with the decrease of $\sigma$. In similarity to the $\sigma-\mathrm{CP}$ the pair mean-field approximation also gives first and second order transitions, with the tricriti- 

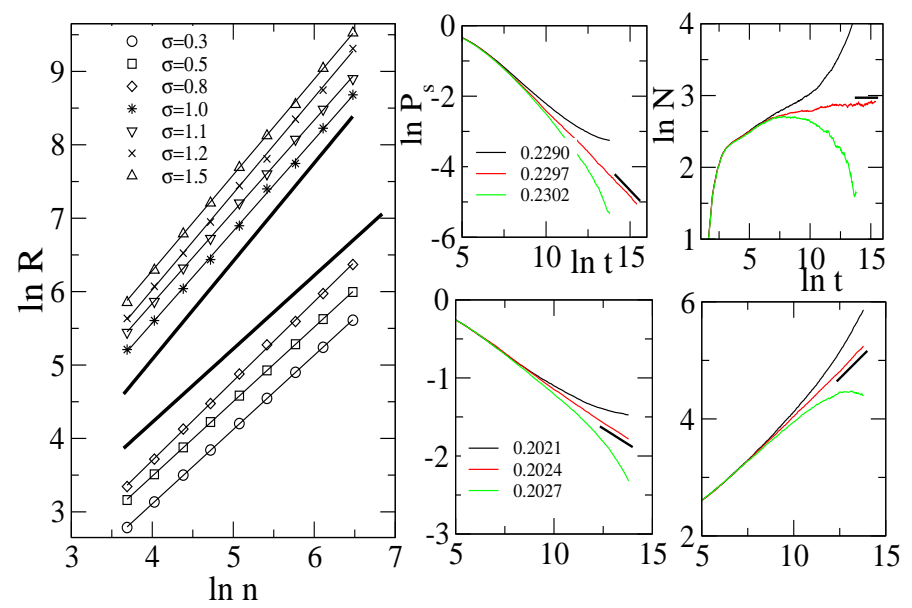

FIG. 6: Log-log plot of $R$ versus $n$ for the long-range PAM and distinct values of $\sigma$. Straight lines have slopes 1.33704 (up) and 1 (down), respectively and they have been shifted in order to avoid overlapping. In the right, from top to bottom, $\log$-log plot of the time evolution of $P_{s}$ and $N$ for distinct $\alpha$ 's for $\sigma=0.3$ (top) and $\sigma=1.2$ (bottom), respectively. The predicted asymptotic slopes are consistent with GI (top) and DP (bottom) values.

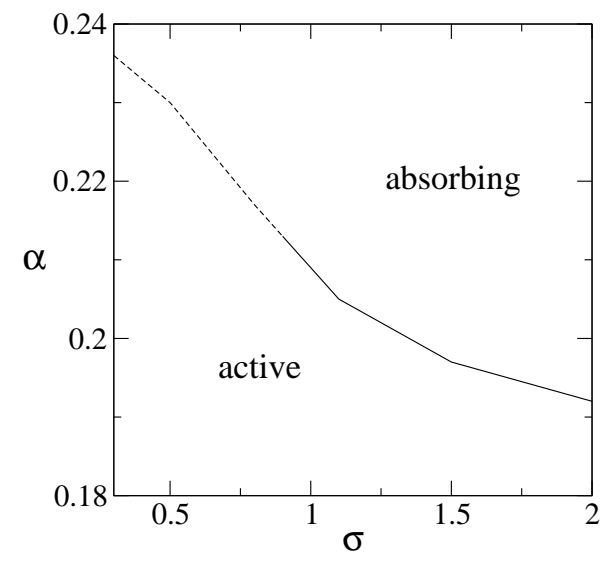

FIG. 7: Parameter $\alpha$ versus $\sigma$ for the long-range PAM. Continuous and dashed lines denote second and first-order transitions, respectively.

cal point given by

$$
\zeta\left(\sigma_{t}\right)=4 \frac{1+a}{a} .
$$

Again, from this equation it follows that $\sigma_{t}>1$. The pair mean-field predicts a critical line at $\alpha_{c}=1 / 2$.

Now we consider the influence of annihilation of three adjacent particles. This study is motivated by previous works 27, 32] which shows that the inclusion of triplet annihilation brings great differences in the phase diagram, when compared with single and pair annihilations. The transition rate $\omega_{i}^{a}$ is then given by

$$
\omega_{i}^{a}=\eta_{i-1} \eta_{i} \eta_{i+1},
$$
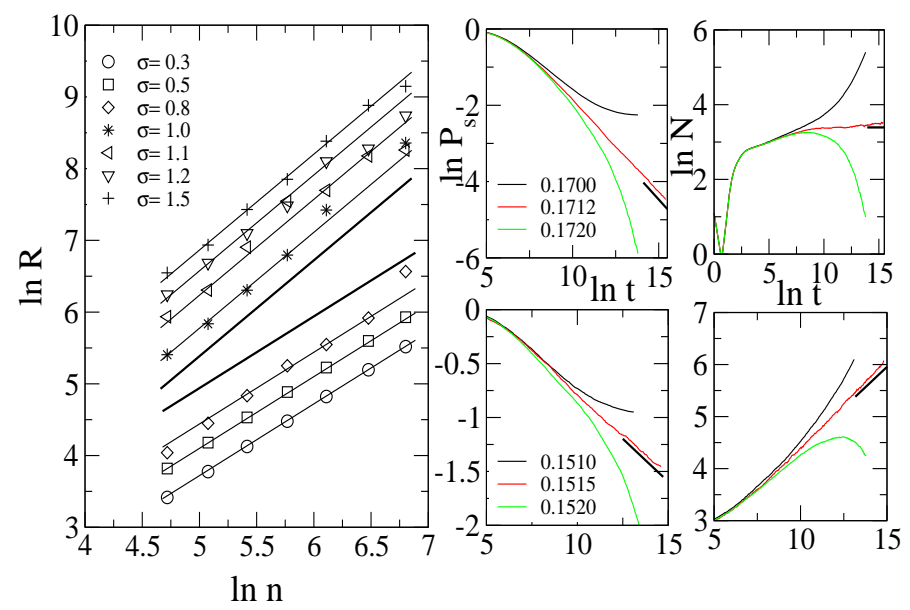

FIG. 8: Log-log plot of $R$ versus $n$ for the long-range TAM and distinct values of $\sigma$. Straight lines have slopes 1.337 (up) and 1 (down), respectively and they have been shifted in order to avoid overlapping. In the right, from top to bottom, loglog plot of the time evolution of $P_{s}$ and $N$ for distinct values of $\alpha$ for $\sigma=0.3$ (top) and $\sigma=1.2$ (bottom), respectively. The predicted asymptotic slopes in the top and bottom are consistent with GI and DP values.

and particles are created like the $\sigma-\mathrm{CP}$. In the conserved ensemble, Eq. (4) is used for calculating for $\bar{\alpha}$ for $k=3$. The short-range case $(a=0)$ has been extensively studied at Refs. [27, 32], where a continuous phase transition belonging to the DP universality class takes place at $\alpha_{c}=0.14898(5)$. In Figs. 8 and 9, we plot the average cluster size $R$ versus $n$ and the phase diagram for different values of $\sigma$. As in previous cases, the phase transition is second-order for $\sigma>1$, becoming first-order for $0<\sigma<1$. The crossover between continuous and discontinuous takes place between 0.8 and 1.1. As an effect of the triplet annihilation, the compact clusters are less dense than all previous cases (for $a=2$ ).

Using the same procedure adopted previously, we have built the phase diagram shown in Fig. 7. The transition points $\alpha$ varies mildly with $\sigma$, as an effect of simultaneous increase of $\left\langle\omega_{i}^{c}\right\rangle_{c}$ and $\left\langle\omega_{i}^{a}\right\rangle_{c}$ by decreasing $\sigma$. For smaller $a$, the system also presents a phase coexistence, although the crossover seems occur for smaller $\sigma$, when compared with the case $a=2$. For example, for $a=0.1$ and $\sigma=0.1$, the phase coexistence yields at $\alpha_{0}=0.1558(1)$.

As in the previous cases, the pair mean-field approximation reproduces first- and second-order transitions, with a critical line and tricritical point occurring at $\alpha_{c}=2 / 3$ and

$$
\zeta\left(\sigma_{t}\right)=9 \frac{1+a}{a},
$$

respectively. Also, as in previous cases, it follows that $\sigma_{t}>1$. In summary, the existence of a discontinuous transition in both PAM and TAM for $0<\sigma<1$ indicate that the pair and triplet annihilations does not provoke sufficient strong fluctuations that would lead the suppression of compact clusters. 


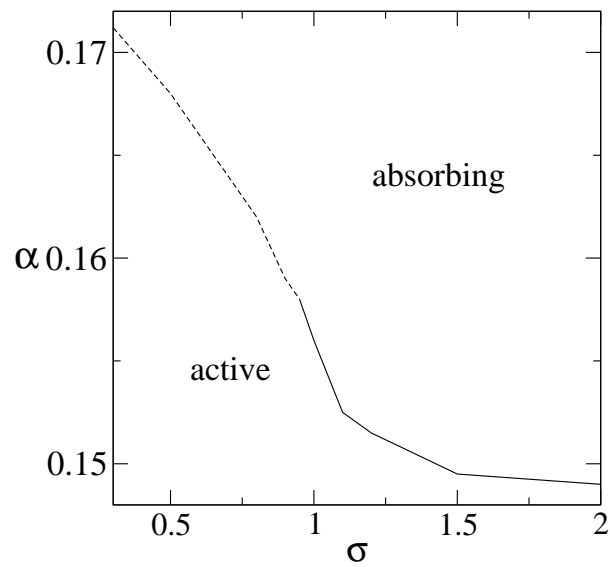

FIG. 9: Parameter $\alpha$ versus $\sigma$ for the long-range TAM. Continuous and dashed lines denote second and first-order transitions, respectively.

\section{Long-range pair contact process $(\sigma-\mathbf{P C P})$}

In the pair contact process (PCP), only pairs of particles can be annihilated or creating new particles. Unlike all previous models, any configuration devoid of pairs is absorbing and thus the PCP displays infinitely many absorbing states. The order parameter is pair density $\phi$ instead of the particle density $\rho$. The PCP model has been extensively studied in the last years [28, 32] and despite the differences with the all previous models, its absorbing transition belongs to the DP universality class. Being $p$ the probability of annihilating pairs of particles, the phase transition yields at at $p_{c}=0.077090(5)$ 28, 32]. The parameters $p$ and $\alpha$ (used here) are related through expression $p=\frac{\alpha}{\alpha+1}$.

The long range version can be introduced similarly than all previous cases. However, in order to investigate the role of infinitely absorbing states, we take two different cases. In the former, the activation rate is given by Eq. (15), implying that the distance $\ell$ is measured up to the nearest particle at the edge of an inactive island. The latter takes into account the distance up to the nearest pair given by

$$
\begin{aligned}
& \omega_{i}^{c}=\frac{1}{2} \sum_{\ell=1}^{\infty}\left(1+\frac{a}{\ell^{\sigma}}\right) \eta_{i-2} \eta_{i-1} \bar{\eta}_{i} \ldots \eta_{i+\ell} \eta_{i+\ell+1} \\
& +\frac{1}{2} \sum_{\ell=1}^{\infty}\left(1+\frac{a}{\ell^{\sigma}}\right) \eta_{i+2} \eta_{i+1} \bar{\eta}_{i} \ldots \eta_{i-\ell-1} \eta_{i-\ell} .
\end{aligned}
$$

In both cases, the annihilation rate is given by Eq. (16).

Since the existence of infinite absorbing states makes the use of spreading simulations difficult, we adopt the procedure described in Sec. II, consisting of studying the time evolution of the pair density $\phi$ starting from a fully occupied lattice. In Fig. 10 we plot the decay of $\phi$ for different $\sigma$ 's by taking the first version. We focus the
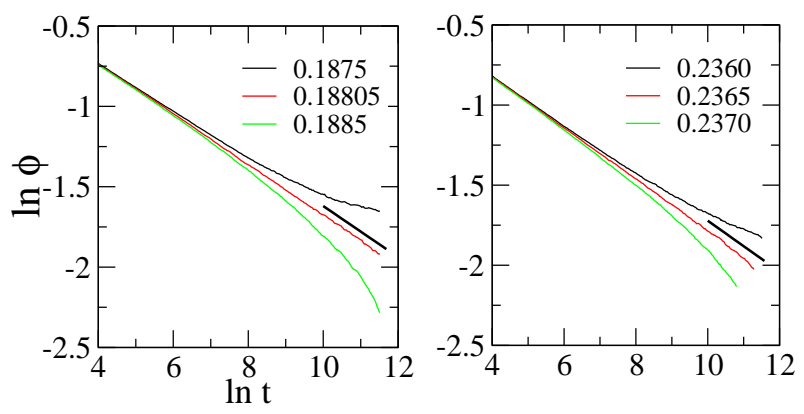

FIG. 10: From the left to right, log-log plot of the time evolution of pair density $\phi$ starting from a fully occupied lattice for $\sigma=0.5$ and $\sigma=0.1$ for different $\alpha$ 's. The straight lines in the middle curves have slopes consistent with $0.159464(6)$.

analysis for $a=2$ and low $\sigma$, in order to see the effect of strong long-range interactions. We see that for both cases, the decay of $\phi$ is algebraic at $0.18805(5)(\sigma=0.5)$ and $0.2365(1)(\sigma=0.1)$ with exponents consistent with the DP value $\theta=0.159464(6)$ (see the black straight lines). This is the first evidence that the phase transition in such case is second-order for all $\sigma$ 's. In order to confirm this query, we also obtained static exponents, by performing steady numerical simulations. In the case of a continuous transition $\phi$ will behave as $\phi \sim\left(\alpha_{c}-\alpha\right)^{\beta}$, where $\beta$ is the associated critical exponent. In the left part of Fig. 11, we show a log-log plot of $\phi$ vs $\Delta \equiv \alpha_{c}-\alpha$ by using the previous estimates for the $\alpha_{c}$ 's. Note that both curves present slopes consistent with the DP value $\beta=0.276486$ (black lines), confirming the second-order phase transition.

We also studied the dependence of the order parameter $\phi$ on the system size $L$. At the criticality $\phi$ decays according to the power law $\phi \sim L^{-\beta / \nu_{\perp}}$, where $\nu_{\perp}$ is the critical exponent associated with the spacial length correlation. In fact, as showed in Fig. 11, for all $\alpha$ 's (circles) $\phi$ also decays algebraically with $L$ with critical exponents consistent with the DP value 0.2520718 (solid lines). Above conclusions remain valid for larger $a$ 's. For example, for $a=5$ and $\sigma=0.5, \phi$ presents an algebraic decay at $\alpha_{c}=0.3318(2)$ with a dynamic exponent consistent with the DP one. The absence of discontinuous transition is understood by inspecting the density of particles surrounded by at least one empty site $\rho-\phi$, which rules the strength of the long-range interaction in such case, as showed in Fig. 11( $d$ ) for $\sigma=0.1$. The existence of infinitely absorbing states leads $\rho-\phi$ to change very mildly (close to the transition), implying that active states with low $\phi$ are not destabilized by the long-range interaction (even for low $\sigma$ ) and hence no abrupt change of $\phi$ occurs and the transition remains continuous.

It is worth mentioning that the absence of discontinuous transition in not predicted by mean-field approximations. By taking two site correlations, the transition is discontinuous for $\sigma<\sigma_{t}$, with a tricritical point separat- 

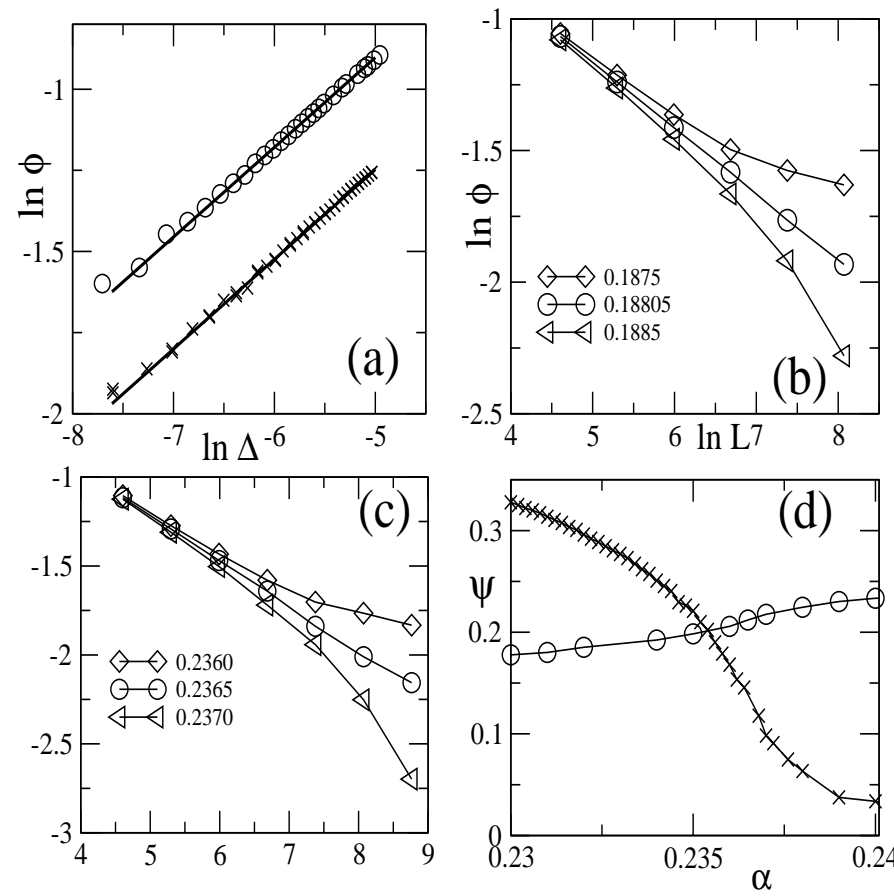

FIG. 11: In $(a)$, the $\log -\log$ plot of $\phi$ versus $\Delta \equiv \alpha_{c}-\alpha$ for different $\sigma$ and $L=3200$. The straight lines have slope 0.276486. Log-log plot of $\phi$ versus $L$ for $\sigma=0.5(b)$ and $\sigma=0.1(c)$. The straight lines have slopes 0.2520718 . In $(d)$ we plot the dependence of $\psi$ on $\alpha$, where $\psi=\phi$ (stars) and $\psi=\rho-\phi$ (circles) for $\sigma=0.1$.

ing first and second lines given by

$$
\zeta\left(\sigma_{t}\right)=2 \frac{1+a}{a} .
$$

Again, from this equation it follows that $\sigma_{t}>1$. The pair mean-field predicts a critical line at $\alpha_{c}=1 / 4$.

In Fig. 12, we show the main results for the second version. In $(a)$ and $(b)$ we compare the time decay of $\phi$ for $a=2$ with $\sigma=1.2$ and $\sigma=0.1$, respectively. In the former case, the slope at $\alpha_{c}=0.09312$ agrees with the value $0.159464(6)$, in consistency with the emergence of second-order transitions for $\sigma>1$. In contrast, the decay for $\sigma=0.1$ is slightly different from the previous case, suggesting a first-order transition. The phase coexistence is confirmed by plotting the pair density probability distribution $P_{\phi}$, as showed in $(c)$ and $(d)$ for $\sigma=1.2$ and $\sigma=0.1$, respectively. In fact, for $\sigma=0.1 P_{\phi}$ is bimodal. Similar results for $\sigma=0.5$ support a first-order transition for $0<\sigma<1$. This result is understood by noting that in the present case $\phi$ plays a similar role to $\rho$ in the $\sigma-\mathrm{CP}$. The dynamics described by Eqs. (16) and (20) allows to relate $\sigma-\mathrm{PCP}$ and the $\sigma-\mathrm{CP}$ through transformation $\eta_{i} \eta_{i+1} \rightarrow \eta_{i}^{\prime}$. Since in the $\sigma$-CP states of low densities are disrupted by the long range interactions, a similar conclusion holds valid the $\sigma$-PCP. For the previous case, such analogy can not be drown, due to the dependence with one site occupied in the frontier
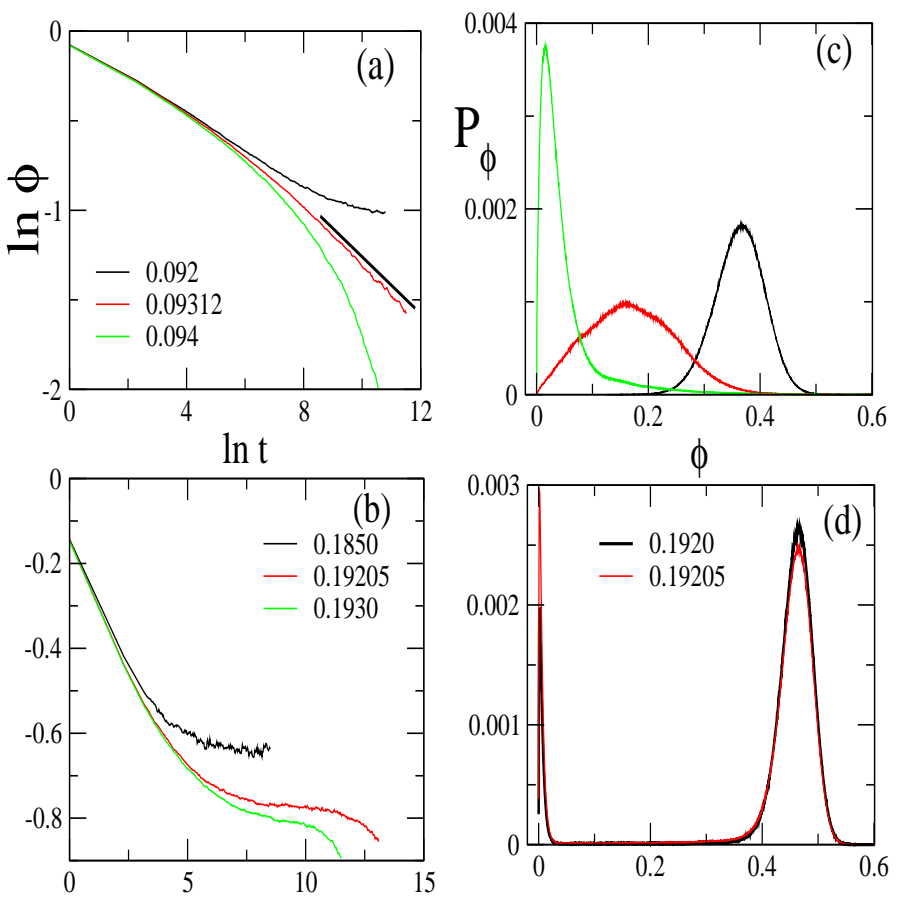

FIG. 12: Log-log plot of the time decay of $\phi$ for $\sigma=1.2$ and $\sigma=0.1$ in $(a)$ and $(b)$, respectively. In $(c)$ and $(d)$, probability distribution $P_{\phi}$ vs $\phi$ for the $\sigma-\mathrm{PCP}$ and $\sigma=1.2$ and $\sigma=0.1$.

instead of two occupied sites. To close this section we remark that the coexistence line also seems to move toward larger $\sigma$ 's when $a$ increases, although the crossover between the two regimes is broader than the $\sigma-\mathrm{CP}$.

\section{CONCLUSION}

First-order phase transitions into absorbing states require a robust mechanism of preventing the creation of particles in low density regimes. Although there are strong evidences that they can not occur in onedimensional short-range contact processes, a long range counterpart by Ginelli et al. 25] revealed such possibility. Aimed at uncovering the fundamental mechanisms ruling long range interactions as an effective mechanism of forming compact clusters, here we investigated thoroughly a family of interaction rules. Our study includes seven contact models grouped in three distinct approaches. In the former, we considered weak long-range interactions and competition between with frequent short-range dynamics, whereas the latter replaces single by cluster interactions. All these results have revealed that it suffices a long-range small perturbation for suppressing low density stable states, in the sense that the undertaken weakening long-range interactions are not sufficient "strong" for destroying compact clusters. The mean-field approach gave us some insight to understand above conclusions. Except the $\sigma$-pair $\mathrm{CP}$, the approximated expressions present a general structure, predicting a changing in the phase 
transition for all $a$ and $\sigma$. However, in contrast with numerical results, the critical lines present the same transition point $\alpha_{c}$ (for all $\sigma>\sigma_{t}$ ). Unlike previous cases, the presence of infinitely many absorbing states leads to novel and different scenarios, depending on the particle structures surrounding the edge of inactive sites. One of them predicts conclusions similar to those of the previous models, whereas in the other version, the phase coexistence is destroyed. Such result can not be understood by the two-site mean-field theory, in which the phase transition also becomes first-order for small values of $\sigma$. Although the increase of fluctuations may predict a second-order transition for small $\sigma$, we believe that, in the present case, a very large order of approximation would be required to reproduce a continuous transition. In sum- mary, long-range interactions constitute an effective dynamics to provide a discontinuous phase transition, even for extreme limits undertaken here. As a final remark, we should mention that the effect of other dynamics such as diffusion and quenched disorder and its competition with long-range interactions should be investigated in a further contribution.

\section{ACKNOWLEDGEMENT}

We acknowledge Miguel A. Muñoz for a critical reading of this manuscript and useful suggestions. The financial support from CNPQ is also acknowledged.
[1] J. Marro and R. Dickman, Nonequilibrium Phase Transitions in Lattice Models (Cambridge University Press, Cambridge, England, 1999).

[2] G. Grinstein and M. A. Muñoz, The Statistical Mechanics of Systems with Absorbing States, in "Fourth Granada Lectures in Computational Physics", edited by P. Garrido and J. Marro, Lecture Notes in Physics, Vol. 493 (Springer, Berlin 1997), p. 223.

[3] T. E. Harris, Ann. Probab. 2, 969 (1974).

[4] H. K. Janssen, Z. Phys. B 42, 151 (1981); P. Grassberger, Z. Phys. B 47, 365 (1982).

[5] M. A. Muñoz, in Advances in Condensed Matter and Statistical Mechanics, edited by E. Korutcheva and R. Cuerno Nova Science, New York, 2004), p. 37.

[6] G. Odor, Rev. Mod. Phys 76, 663 (2004).

[7] K. A. Takeuchi, M. Kuroda, H. Chaté, and M. Sano, Phys. Rev. Lett. 99, 234503 (2007).

[8] See for example, A. Lipowski, Phys. Rev. E 62, 4401 (2000); H. Hinrichsen, Phys. Rev. E 63, 016109 (2000).

[9] Sang-Gui Lee and S. B. Lee, Phys. Rev. E 80, 011106 (2009).

[10] C. E. Fiore and M. J. de Oliveira, Braz. Journ. Phys. 36, 218 (2006).

[11] R. Dickman and T. Tomé, Phys. Rev. A 44, 4833 (1991).

[12] C. E. Fiore and M. J. de Oliveira, Phys. Rev. E 70, 046131 (2004).

[13] G. Cardozo and J. F. Fontanari, Eur. Phys. J. B 51, 555 (2006).

[14] H. Hinrichsen, arXiv:cond-mat/0006212.

[15] S-C. Park, Phys. Rev. E 80, 061103 (2009).

[16] D. Vernon and M. Howard, Phys. Rev. E 63, 041116 (2001)

[17] D. C. Vernon, Phys. Rev. E 68, 041103 (2003).

[18] D. Mollison, J. R. Stat. Soc. B 39, 283 (1977).

[19] P. Grassberger, in Fractals in Physics, edited by L.
Pietronero and E. Tosatti, (Elsevier, New York, 1986).

[20] H. K. Janssen, K. Oerding, F. van Wijland and H. J. Hilhorst, Eur. Phys. J. B 7, 137 (1999).

[21] H. Hinrichsen and M. Howard, Eur. J. Phys. B 7635 (1999).

[22] C. J. Tessone, M. Cencini and A. Torcini, Phys. Rev. Lett. 97, 224101 (2006).

[23] F.Ginelli, H. Hinrichsen, R. Livi, D. Mukamel and A. Torcini, J. Stat. Mech. P08008 (2006).

[24] H. Hinrichsen, J. Stat. Mech. P07066 (2007).

[25] F.Ginelli, H. Hinrichsen, R. Livi, D. Mukamel and A. Politi, Phys. Rev. E 71, 026121 (2005).

[26] C. E. Fiore and M. J. de Oliveira, Phys. Rev. E 76, 041103 (2007).

[27] R. Dickman, Phys. Rev. B 40, 7005 (1989).

[28] I. Jensen, Phys. Rev. Lett. 70, 1465 (1993).

[29] T. Tomé and M. J. de Oliveira, Phys. Rev. Lett. 86, 5643 (2001).

[30] H. J. Hilhorst and F. van Wijland, Phys. Rev. E 65, 035103(R) (2002).

[31] M. J. de Oliveira, Phys. Rev. E 67, 027104 (2003).

[32] C. E. Fiore and M. J. de Oliveira, Phys. Rev. E 72, 046137 (2005).

[33] I. Jensen and R. Dickman, Phys. Rev. E 48, 1710 (1993).

[34] M. A. Muñoz, G. Grinstein, R. Dickman and R. Livi, Phys. Rev. E 76, 451 (1995).

[35] M. A. Muñoz, G. Grinstein, R. Dickman and R. Livi, Physica D 103, 485 (1997).

[36] M. A. Muñoz, G. Grinstein and R. Dickman, J. Stat. Phys. 91, 541 (1998).

[37] H.-M. Bröker and P. Grassberger, Physica A 267, 453 (1999).

[38] T. Vicsek, Fractal Growth Phenomena, 2nd ed. (World Scientific, Singapoure, 1992). 